Bull. Korean Math. Soc. 49 (2012), No. 6, pp. 1263-1274

http://dx.doi.org/10.4134/BKMS.2012.49.6.1263

\title{
ON OPIAL INEQUALITIES INVOLVING HIGHER ORDER DERIVATIVES
}

\author{
Chang-Jian ZhaO And Wing-Sum Cheung
}

\begin{abstract}
In the present paper we establish some new Opial-type inequalities involving higher order partial derivatives. The results in special cases yield some of the recent results on Opial's inequality and provide new estimates on inequalities of this type.
\end{abstract}

\section{Introduction}

In the year 1960, Opial [21] established the following inequality:

Theorem A. Suppose $f \in C^{1}[0, a]$ satisfies $f(0)=f(a)=0$ and $f(x)>0$ for all $x \in(0, a)$. Then the inequality holds

$$
\int_{0}^{a}\left|f(x) f^{\prime}(x)\right| d x \leq \frac{a}{4} \int_{0}^{a}\left(f^{\prime}(x)\right)^{2} d x,
$$

where this constant a/4 is best possible.

Opial's inequality and its generalizations, extensions and discretizations play a fundamental role in establishing the existence and uniqueness of initial and boundary value problems for ordinary and partial differential equations as well as difference equations $[2,3,7,18,20]$. The inequality (1.1) has received considerable attention and a large number of papers dealing with new proofs, extensions, generalizations, variants and discrete analogues of Opial's inequality have appeared in the literature [9-13,15, 19, 22-29,31].

The first natural extension of Opial's inequality (1.1) involving $x^{(n)}(t), n \geq 1$ instead of $x^{\prime}(t)$ is embodied in the following:

Theorem B ([30]). Let $x(t) \in C^{n}[0, a]$ be such that $x^{(i)}(0)=0,0 \leq i \leq n-1$. Then the inequality holds

$$
\int_{0}^{a}\left|x(t) x^{(n)}(t)\right| d t \leq \frac{1}{2} a^{n} \int_{0}^{a}\left|x^{(n)}(t)\right|^{2} d t .
$$

Received February 19, 2011; Revised December 19, 2011.

2010 Mathematics Subject Classification. 26D15.

Key words and phrases. Opial's inequality, Opial-type inequalities, Hölder's inequality. The first author is supported by National Natural Science Foundation of China(10971205).

The second author is partially supported by a HKU URG grant. 
A sharper version of (1.2) is the following:

Theorem C ([14]). Let $x(t) \in C^{n-1}[0, a]$ be such that $x^{(i)}(0)=0,0 \leq i \leq n-1$. Further, let $x^{(n-1)}(t)$ be absolutely continuous, and $\int_{0}^{n}\left|x^{(n)}(t)\right|^{2} d t<\infty$. Then the inequality holds

$$
\int_{0}^{a}\left|x(t) x^{(n)}(t)\right| d t \leq c_{n} a^{n} \int_{0}^{a}\left|x^{(n)}(t)\right|^{2} d t
$$

with equality if and only if $n=1$ and $x^{(n)}(t)=c$, where $c_{n}=\frac{1}{2 n !}\left(\frac{n}{2 n-1}\right)^{\frac{1}{2}}$.

A result involving two functions and their higher order derivatives is embodied in the following:

Theorem D $([23])$. For $j=1,2$, let $x_{j}(t) \in C^{n-1}[0, a]$ be such that $x_{j}^{(i)}(0)=$ $0,0 \leq i \leq n-1$. Further, let $x_{j}^{(n-1)}(t)$ be absolutely continuous, and

$$
\int_{0}^{n}\left|x_{j}^{(n)}(t)\right|^{2} d t<\infty
$$

Then the inequality holds

$\int_{0}^{a}\left(\left|x_{1}(t) \cdot x_{2}^{(n)}(t)\right|+\left|x_{2}(t) \cdot x_{1}^{(n)}(t)\right|\right) d t \leq c_{n} a^{n} \int_{0}^{a}\left(\left|x_{1}^{(n)}(t)\right|^{2}+\left|x_{2}^{(n)}(t)\right|^{2}\right) d t$,

with equality if and only if $n=1, x_{j}^{(n)}(t)=c$, where the constant $c_{n}$ is defined in Theorem $C$.

For Opial type integral inequalities involving high-order partial derivatives see $[1,4,6,17,33]$. For an extensive survey on these inequalities, see [3]. The first aim of this paper is to establish the following Opial-type inequality involving two functions and their higher order partial derivatives based on applications of improvements of Das [14] and Pachpatte's ideas [23].

Theorem 1.1. Let $a, b, s, t, \sigma, \tau$ be real numbers, $0 \leq \sigma \leq s \leq a$ and $0 \leq \tau \leq$ $t \leq b$. For $j=1,2, n \geq 1, k \geq 1$, let $x_{j}(s, t) \in C^{(n-1)}[0, a] \times C^{(k-1)}[0, b]$ be such that $\frac{\partial^{i}}{\partial \sigma^{i}} x_{j}(0, \tau)=0, \frac{\partial^{i^{\prime}}}{\partial \tau^{i^{\prime}}} x_{j}(\sigma, 0)=0, \sigma \in[0, s], \tau \in[0, t], 0 \leq i \leq n-1,0 \leq$ $i^{\prime} \leq k-1$, and $x_{j}^{(n, k)}(s, \tau)=0, x_{j}^{(n, k)}(\sigma, t)=0$. Further, let $x_{j}^{(n-1, k-1)}(s, t)$ be absolutely continuous, and $\int_{0}^{a} \int_{0}^{b}\left|x_{j}^{(n, k)}(s, t)\right|^{2} d s d t<\infty$. Then

$$
\begin{aligned}
& \int_{0}^{a} \int_{0}^{b}\left(\left|x_{1}(s, t) \cdot x_{2}^{(n, k)}(s, t)\right|+\left|x_{2}(s, t) \cdot x_{1}^{(n, k)}(s, t)\right|\right) d s d t \\
\leq & c_{n, k} a^{n} b^{k} \int_{0}^{a} \int_{0}^{b}\left(\left|x_{1}^{(n, k)}(s, t)\right|^{2}+\left|x_{2}^{(n, k)}(s, t)\right|^{2}\right) d s d t,
\end{aligned}
$$


with equality if and only if $n=k=1, x_{j}^{(n, k)}(s, t)=c$, where

$$
x_{j}^{(n, k)}(s, t)=\frac{\partial^{n}}{\partial s^{n}}\left(\frac{\partial^{k}}{\partial t^{k}} x_{j}(s, t)\right),
$$

and

$$
c_{n, k}=\frac{1}{4 n ! k !}\left(\frac{2 n k}{(2 n-1)(2 k-1)}\right)^{\frac{1}{2}} .
$$

We also prove an interesting Opial-type inequality involving two functions and their higher order partial derivatives as follows:

Theorem 1.2. Let $a, b, s, t, \sigma, \tau$ be real numbers, $0 \leq \sigma \leq s \leq a$ and $0 \leq \tau \leq$ $t \leq b$. Let $l>0,0<m<1$ and satisfying $l+m>1$. For $j=1,2, n \geq$ $1, \bar{k} \geq 1$, let $x_{j}(s, t) \in C^{(n-1)}[0, a] \times C^{(k-1)}[0, b]$ be such that $\frac{\partial^{i}}{\partial \sigma^{i}} x_{j}(0, \tau)=$ $0, \frac{\partial^{i^{\prime}}}{\partial \tau^{i^{\prime}}} x_{j}(\sigma, 0)=0, \sigma \in[0, s], \tau \in[0, t], 0 \leq i \leq n-1,0 \leq i^{\prime} \leq k-1$. Further, let $x_{j}^{(n-1, k-1)}(s, t)$ be absolutely continuous, and $\int_{0}^{a} \int_{0}^{b}\left|x_{j}^{(n, k)}(s, t)\right|^{l+m} d s d t<\infty$. Then

$$
\begin{aligned}
& \int_{0}^{a} \int_{0}^{b}\left[\left|x_{1}(s, t)\right|^{l}\left|x_{2}^{(n, k)}(s, t)\right|^{m}+\left|x_{2}(s, t)\right|^{l}\left|x_{1}^{(n, k)}(s, t)\right|^{m}\right] d s d t \\
\leq & c_{n, k}^{*} a^{n l} b^{k l}\left[\left(\int_{0}^{a} \int_{0}^{b}\left|x_{1}^{(n, k)}(s, t)\right|^{l+m} d s d t\right)^{\frac{l}{m}} \cdot \int_{0}^{a} \int_{0}^{b}\left|x_{2}^{(n, k)}(s, t)\right|^{m+l} d s d t\right. \\
& \left.+\left(\int_{0}^{a} \int_{0}^{b}\left|x_{2}^{(n, k)}(s, t)\right|^{l+m} d s d t\right)^{\frac{l}{m}} \cdot \int_{0}^{a} \int_{0}^{b}\left|x_{1}^{(n, k)}(s, t)\right|^{m+l} d s d t\right]^{\frac{m}{l+m}},
\end{aligned}
$$

where

$$
c_{n, k}^{*}=\left(\frac{n k(1-\xi)^{2}}{(n-\xi)(k-\xi)}\right)^{l(1-\xi)} \frac{2^{\xi l} \xi^{2 \xi l}}{(n ! k !)^{l}}, \quad \xi=\frac{1}{l+m} .
$$

\section{Main results and their proofs}

Our main results are given in the following theorems.

Theorem 2.1. Let $a, b, s, t, \sigma, \tau$ be real numbers, $0 \leq \sigma \leq s \leq a$ and $0 \leq \tau \leq$ $t \leq b$. For $j=1,2, n \geq 1, k \geq 1$, let $x_{j}(s, t) \in C^{(n-1)}[0, a] \times C^{(k-1)}[0, b]$ be such that $\frac{\partial^{i}}{\partial \sigma^{i}} x_{j}(0, \tau)=0, \frac{\partial^{i^{\prime}}}{\partial \tau^{i^{\prime}}} x_{j}(\sigma, 0)=0, \sigma \in[0, s], \tau \in[0, t], 0 \leq i \leq n-1,0 \leq$ $i^{\prime} \leq k-1$, and $x_{j}^{(n, k)}(s, \tau)=0, x_{j}^{(n, k)}(\sigma, t)=0$. Further, let $x_{j}^{(n-1, k-1)}(s, t)$ be 
absolutely continuous, and $\int_{0}^{a} \int_{0}^{b}\left|x_{j}^{(n, k)}(s, t)\right|^{2} d s d t<\infty$. Then

$$
\begin{aligned}
& \int_{0}^{a} \int_{0}^{b}\left(\left|x_{1}(s, t) \cdot x_{2}^{(n, k)}(s, t)\right|+\left|x_{2}(s, t) \cdot x_{1}^{(n, k)}(s, t)\right|\right) d s d t \\
\leq & c_{n, k} a^{n} b^{k} \int_{0}^{a} \int_{0}^{b}\left(\left|x_{1}^{(n, k)}(s, t)\right|^{2}+\left|x_{2}^{(n, k)}(s, t)\right|^{2}\right) d s d t,
\end{aligned}
$$

with equality if and only if $n=k=1, x_{j}^{(n, k)}(s, t)=c$, where $x_{j}^{(n, k)}(s, t)$ and $c_{n, k}$ are defined in Theorem 1.1, respectively.

Proof. For $\sigma$, integration by parts $(n-1)$-times in right side of the following first formula and in view of $\frac{\partial^{i}}{\partial \sigma^{i}} x(0, \tau)=0, \frac{\partial^{i^{\prime}}}{\partial \tau^{i^{\prime}}} x(\sigma, 0)=0,0 \leq i \leq n-1,0 \leq$ $i^{\prime} \leq k-1$, we have

$$
\begin{aligned}
& x_{1}(s, t) \\
= & \frac{(-1)^{n}}{(n-1) !} \int_{s}^{0}(\sigma-s)^{n-1} \frac{\partial^{n}}{\partial \sigma^{n}} x_{1}(\sigma, t) d \sigma \\
= & \frac{(-1)^{2 n-1}}{(n-1) !} \int_{s}^{0}(s-\sigma)^{n-1} \frac{\partial^{n}}{\partial \sigma^{n}} x_{1}(\sigma, t) d \sigma \\
= & \frac{1}{(n-1) !} \int_{0}^{s}(s-\sigma)^{n-1} \frac{\partial^{n}}{\partial \sigma^{n}} x_{1}(\sigma, t) d \sigma \\
= & \frac{1}{(n-1) !(k-1) !} \int_{0}^{s}(s-\sigma)^{n-1} \frac{\partial^{n}}{\partial \sigma^{n}}\left(\int_{0}^{t}(t-\tau)^{k-1} \frac{\partial^{k}}{\partial \tau^{k}} x_{1}(\sigma, \tau) d \tau\right) d \sigma \\
= & \frac{1}{(n-1) !(k-1) !} \int_{0}^{s} \int_{0}^{t}(s-\sigma)^{n-1}(t-\tau)^{k-1} \cdot x_{1}^{(n, k)}(\sigma, \tau) d \sigma d \tau .
\end{aligned}
$$

Multiplying both sides of $(2.2)$ by $x_{2}^{(n, k)}(s, t)$ and using the Cauchy-Schwarz inequality, we have

$$
\begin{aligned}
& \left|x_{1}(s, t) \cdot x_{2}^{(n, k)}(s, t)\right| \\
\leq & \frac{\left|x_{2}^{(n, k)}(s, t)\right|}{(n-1) !(k-1) !}\left(\int_{0}^{s} \int_{0}^{t}(s-\sigma)^{2 n-2}(t-\tau)^{2 k-2} d \sigma d \tau\right)^{\frac{1}{2}} \\
& \times\left(\int_{0}^{s} \int_{0}^{t}\left|x_{1}^{(n, k)}(\sigma, \tau)\right|^{2} d \sigma d \tau\right)^{\frac{1}{2}} \\
= & \frac{1}{(n-1) !(k-1) ! \sqrt{(2 n-1)(2 k-1)}} \cdot s^{n-\frac{1}{2}} t^{k-\frac{1}{2}}\left|x_{2}^{(n, k)}(s, t)\right| \\
& \times\left(\int_{0}^{s} \int_{0}^{t}\left|x_{1}^{(n, k)}(\sigma, \tau)\right|^{2} d \sigma d \tau\right)^{\frac{1}{2}} \cdot
\end{aligned}
$$

Thus, integrating both sides of (2.3) over $t$ from 0 to $b$ first and then integrating the resulting inequality over $s$ from 0 to $a$ and applying the Cauchy-Schwarz 
ON OPIAL INEQUALITIES INVOLVING HIGHER ORDER DERIVATIVES

1267

inequality again, we obtain

$$
\begin{aligned}
& \int_{0}^{a} \int_{0}^{b}\left|x_{1}(s, t) \cdot x_{2}^{(n, k)}(s, t)\right| d s d t \\
\leq & \frac{1}{(n-1) !(k-1) ! \sqrt{(2 n-1)(2 k-1)}}\left(\int_{0}^{a} \int_{0}^{b} s^{2 n-1} t^{2 k-1} d s d t\right)^{\frac{1}{2}} \\
& \times\left(\int_{0}^{a} \int_{0}^{b}\left|x_{2}^{(n, k)}(s, t)\right|^{2}\left(\int_{0}^{s} \int_{0}^{t}\left|x_{1}^{(n, k)}(\sigma, \tau)\right|^{2} d \sigma d \tau\right) d s d t\right)^{\frac{1}{2}} \\
= & \frac{1}{2 n !}\left(\frac{2 n}{2 n-1}\right)^{\frac{1}{2}} \frac{1}{2 k !}\left(\frac{2 k}{2 k-1}\right)^{\frac{1}{2}} a^{n} b^{k} \\
& \times\left(\int_{0}^{a} \int_{0}^{b}\left|x_{2}^{(n, k)}(s, t)\right|^{2}\left(\int_{0}^{t} \int_{0}^{t}\left|x_{1}^{(n, k)}(\sigma, \tau)\right|^{2} d \sigma d \tau\right) d s d t\right)^{\frac{1}{2}} .
\end{aligned}
$$

Similarly, we obtain

$$
\begin{aligned}
& \int_{0}^{a} \int_{0}^{b}\left|x_{2}(s, t) \cdot x_{1}^{(n, k)}(s, t)\right| d s d t \\
\leq & \frac{1}{2 n !}\left(\frac{2 n}{2 n-1}\right)^{\frac{1}{2}} \frac{1}{2 k !}\left(\frac{2 k}{2 k-1}\right)^{\frac{1}{2}} a^{n} b^{k} \\
& \times\left(\int_{0}^{a} \int_{0}^{b}\left|x_{1}^{(n, k)}(s, t)\right|^{2}\left(\int_{0}^{s} \int_{0}^{t}\left|x_{2}^{(n, k)}(\sigma, \tau)\right|^{2} d \sigma d \tau\right) d s d t\right)^{\frac{1}{2}} .
\end{aligned}
$$

Thus, from $(2.4),(2.5)$ and in view of the elementary inequality $a^{\frac{1}{2}}+b^{\frac{1}{2}} \leq$ $[2(a+b)]^{\frac{1}{2}}, a, b \geq 0$, we obtain

$$
\begin{aligned}
& \int_{0}^{a} \int_{0}^{b}\left(\left|x_{1}(s, t) \cdot x_{2}^{(n, k)}(s, t)\right|+\left|x_{2}(s, t) \cdot x_{1}^{(n, k)}(s, t)\right|\right) d s d t \\
\leq & 2 c_{n, k} a^{n} b^{k}\left[\int _ { 0 } ^ { a } \int _ { 0 } ^ { b } \left\{\left|x_{2}^{(n, k)}(s, t)\right|^{2}\left(\int_{0}^{s} \int_{0}^{t}\left|x_{1}^{(n, k)}(\sigma, \tau)\right|^{2} d \sigma d \tau\right)\right.\right. \\
+ & \left.\left.\left|x_{1}^{(n, k)}(s, t)\right|^{2}\left(\int_{0}^{s} \int_{0}^{t}\left|x_{2}^{(n, k)}(\sigma, \tau)\right|^{2} d \sigma d \tau\right)\right\} d s d t\right]^{\frac{1}{2}} .
\end{aligned}
$$

On the other hand

$$
\begin{aligned}
& \frac{\partial^{2}}{\partial s \partial t} {\left[\left(\int_{0}^{s} \int_{0}^{t}\left|x_{1}^{(n, k)}(\sigma, \tau)\right|^{2} d \sigma d \tau\right)\left(\int_{0}^{s} \int_{0}^{t}\left|x_{2}^{(n, k)}(\sigma, \tau)\right|^{2} d \sigma d \tau\right)\right] } \\
&=\frac{\partial}{\partial s}\left[\int_{0}^{s}\left|x_{1}(\sigma, t)\right|^{2} d \sigma \cdot \int_{0}^{s} \int_{0}^{t}\left|x_{2}^{(n, k)}(\sigma, \tau)\right|^{2} d \sigma d \tau\right. \\
&\left.+\int_{0}^{s} \int_{0}^{t}\left|x_{1}^{(n, k)}(\sigma, \tau)\right|^{2} d \sigma d \tau \cdot \int_{0}^{s}\left|x_{2}(\sigma, t)\right|^{2} d \sigma\right]
\end{aligned}
$$




$$
\begin{aligned}
= & \left|x_{1}^{(n, k)}(s, t)\right|^{2} \cdot \int_{0}^{s} \int_{0}^{t}\left|x_{2}^{(n, k)}(\sigma, \tau)\right|^{2} d \sigma d \tau \\
& +\int_{0}^{s}\left|x_{1}(\sigma, t)\right|^{2} d \sigma \cdot \int_{0}^{t}\left|x_{2}(s, \tau)\right|^{2} d \tau \\
& +\left|x_{2}^{(n, k)}(s, t)\right|^{2} \cdot \int_{0}^{s} \int_{0}^{t}\left|x_{1}^{(n, k)}(\sigma, \tau)\right|^{2} d \sigma d \tau \\
& +\int_{0}^{s}\left|x_{2}(\sigma, t)\right|^{2} d \sigma \cdot \int_{0}^{t}\left|x_{1}(s, \tau)\right|^{2} d \tau \\
= & \left|x_{1}^{(n, k)}(s, t)\right|^{2} \cdot \int_{0}^{s} \int_{0}^{t}\left|x_{2}^{(n, k)}(\sigma, \tau)\right|^{2} d \sigma d \tau \\
& +\left|x_{2}^{(n, k)}(s, t)\right|^{2} \cdot \int_{0}^{s} \int_{0}^{t}\left|x_{1}^{(n, k)}(\sigma, \tau)\right|^{2} d \sigma d \tau .
\end{aligned}
$$

From $(2.6),(2.7)$ and in view of the elementary inequality $2(a b)^{\frac{1}{2}} \leq a+b, a, b \geq$ 0 , we obtain,

$$
\begin{aligned}
\int_{0}^{a} \int_{0}^{b}\left(\left|x_{1}(s, t) \cdot x_{2}^{(n, k)}(s, t)\right|+\left|x_{2}(s, t) \cdot x_{1}^{(n, k)}(s, t)\right|\right) d s d t \\
\leq 2 c_{n, k} a^{n} b^{k}\left[\int _ { 0 } ^ { a } \int _ { 0 } ^ { b } \frac { \partial ^ { 2 } } { \partial s \partial t } \left\{\left(\int_{0}^{s} \int_{0}^{t}\left|x_{1}^{(n, k)}(\sigma, \tau)\right|^{2} d \sigma d \tau\right)\right.\right. \\
\left.\left.\times\left(\int_{0}^{s} \int_{0}^{t}\left|x_{2}^{(n, k)}(\sigma, \tau)\right|^{2} d \sigma d \tau\right)\right\} d s d t\right]^{\frac{1}{2}} \\
=2 c_{n, k} a^{n} b^{k}\left[\int_{0}^{a} \int_{0}^{b}\left|x_{1}^{(n, k)}(s, t)\right|^{2} d s d t \cdot \int_{0}^{a} \int_{0}^{b}\left|x_{2}^{(n, k)}(s, t)\right|^{2} d s d t\right]^{\frac{1}{2}} \\
\leq c_{n, k} a^{n} b^{k} \int_{0}^{a} \int_{0}^{b}\left(\left|x_{1}^{(n, k)}(s, t)\right|^{2}+\left|x_{2}^{(n, k)}(s, t)\right|^{2}\right) d s d t .
\end{aligned}
$$

Remark 2.2. Let $x(s, t)$ reduce to $x(t)$ by letting $s=s(t)$ and with suitable modifications in all intermediate steps of proof of Theorem 2.1, then Theorem 2.1 becomes Theorem D stated in the introduction which was established by Pachpatte [23].

Remark 2.3. Taking for $x_{1}(s, t)=x_{2}(s, t)=x(s, t)$ in $(2.1)$, we have

$$
\int_{0}^{a} \int_{0}^{b}\left|x(s, t) \cdot x^{(n, k)}(s, t)\right| d s d t \leq c_{n, k} a^{n} b^{k} \int_{0}^{a} \int_{0}^{b}\left|x^{(n, k)}(s, t)\right|^{2} d s d t
$$

with equality if and only if $n=1, x^{(n)}(s, t)=c$. 
Let $x(s, t)$ reduce to $x(t)$ by letting $s=s(t)$ and with suitable modifications, then (2.8) becomes the following inequality:

$$
\int_{0}^{a}\left|x(t) \cdot x^{(n)}(t)\right| d t \leq c_{n} a^{n} \int_{0}^{a}\left|x^{(n)}(t)\right|^{2} d t,
$$

with equality if and only if $n=1, x^{(n)}(t)=c$, where the constant $c_{n}$ is defined in (1.3).

This is just an inequality in Theorem $\mathrm{C}$ stated in the introduction established by Das [14].

Remark 2.4. Let $0 \leq \alpha, \beta<n$, for $j=1,2$ let $g_{j}(s, t) \in C^{(n-\alpha-1)}[0, a] \times$ $C^{(k-\beta-1)}[0, b]$ be such that $\frac{\partial^{i}}{\partial s^{i}} g_{j}(0, t)=\frac{\partial^{i^{\prime}}}{\partial t^{i^{\prime}}} g_{j}(s, 0)=0,0 \leq i \leq n-\alpha-1,0 \leq$ $i^{\prime} \leq k-\beta-1$ and suppose that $g^{(n-\alpha-1, k-\beta-1)}(s, t)$ are absolutely continuous, and $\int_{0}^{a} \int_{0}^{b}\left|g_{j}^{(n-\alpha, k-\beta)}(s, t)\right|^{2} d s d t<\infty$, then from (2.1) it follows that

$$
\begin{gathered}
\int_{0}^{a} \int_{0}^{b}\left[\left|g_{1}(s, t) \cdot g_{2}^{(n-\alpha, k-\beta)}(s, t)\right|+\left|g_{1}^{(n-\alpha, k-\beta)}(s, t) \cdot g_{2}(s, t)\right|\right] d s d t \\
\leq c_{n-\alpha, k-\beta} a^{n-\alpha} b^{k-\beta} \int_{0}^{a} \int_{0}^{b}\left[\left|g_{1}^{(n-\alpha, k-\beta)}(s, t)\right|^{2}+\left|g_{2}^{(n-\alpha, k-\beta)}(s, t)\right|^{2}\right] d s d t .
\end{gathered}
$$

Thus, for $g_{j}(s, t)=x_{j}^{(\alpha, \beta)}(s, t)$, where $x_{j}(s, t) \in C^{(n-1)}[0, a] \times C^{(k-1)}[0, b]$, $\frac{\partial^{i}}{\partial s^{i}} x(0, t)=0, \frac{\partial^{i^{\prime}}}{\partial t^{i^{\prime}}} x(s, 0)=0, \alpha \leq i \leq n-1, \beta \leq i^{\prime} \leq k-1$, and $x_{j}^{(n-1, k-1)}(s, t)$ are absolutely continuous, and $\int_{0}^{a} \int_{0}^{b}\left|x_{j}^{(n, k)}(s, t)\right|^{2} d s d t<\infty$, then

$$
\begin{aligned}
& \int_{0}^{a} \int_{0}^{b}\left[\left|x_{1}^{(\alpha, \beta)}(s, t) \cdot x_{2}^{(n, k)}(s, t)\right|+\left|x_{1}^{(n, k)}(s, t) \cdot x_{2}^{(\alpha, \beta)}(s, t)\right|\right] d s d t \\
\leq & c_{n-\alpha, k-\beta} a^{n-\alpha} b^{k-\beta} \int_{0}^{a} \int_{0}^{b}\left[\left|x_{1}^{(n, k)}(s, t)\right|^{2}+\left|x_{2}^{(n, k)}(s, t)\right|^{2}\right] d s d t .
\end{aligned}
$$

Obviously, a special case of (2.9) is following inequality:

$$
\begin{aligned}
& \int_{0}^{a} \int_{0}^{b}\left[\left|x_{1}^{(k, k)}(s, t) \cdot x_{2}^{(n, n)}(s, t)\right|+\left|x_{1}^{(n, n)}(s, t) \cdot x_{2}^{(k, k)}(s, t)\right|\right] d s d t \\
\leq & c_{n-k, n-k}(a b)^{n-k} \int_{0}^{a} \int_{0}^{b}\left[\left|x_{1}^{(n, n)}(s, t)\right|^{2}+\left|x_{2}^{(n, n)}(s, t)\right|^{2}\right] d s d t .
\end{aligned}
$$

Let $x_{j}(s, t)$ reduce to $s_{j}(t)$ and with suitable modifications, then $(2.10)$ becomes the following inequality:

$$
\begin{aligned}
& \int_{0}^{a}\left[\left|x_{1}^{(k)}(t) x_{2}^{(n)}(t)\right|+\left|x_{1}^{(n)}(t) x_{2}^{(k)}(t)\right|\right] d t \\
\leq & c_{n-k} a^{n-k} \int_{0}^{a}\left[\left|x_{1}^{(n)}(t)\right|^{2}+\left|x_{1}^{(n)}(t)\right|^{2}\right] d t,
\end{aligned}
$$


where the constant $c_{n-k}$ is defined in (1.3). This is just an new inequality established by Pachpatte [24].

Theorem 2.5. Let $a, b, s, t, \sigma, \tau$ be real numbers, $0 \leq \sigma \leq s \leq a$ and $0 \leq \tau \leq$ $t \leq b$. Let $l>0,0<m<1$ and satisfying $l+m>1$. For $j=1,2, n \geq$ $1, k \geq 1$, let $x_{j}(s, t) \in C^{(n-1)}[0, a] \times C^{(k-1)}[0, b]$ be such that $\frac{\partial^{i}}{\partial \sigma^{i}} x_{j}(0, \tau)=$ $0, \frac{\partial^{i^{\prime}}}{\partial \tau^{i}} x_{j}(\sigma, 0)=0, \sigma \in[0, s], \tau \in[0, t], 0 \leq i \leq n-1,0 \leq i^{\prime} \leq k-1$. Further, let $x_{j}^{(n-1, k-1)}(s, t)$ be absolutely continuous, and $\int_{0}^{a} \int_{0}^{b}\left|x_{j}^{(n, k)}(s, t)\right|^{l+m} d s d t<\infty$. Then

$$
\begin{gathered}
\int_{0}^{a} \int_{0}^{b}\left[\left|x_{1}(s, t)\right|^{l}\left|x_{2}^{(n, k)}(s, t)\right|^{m}+\left|x_{2}(s, t)\right|^{l}\left|x_{1}^{(n, k)}(s, t)\right|^{m}\right] d s d t \\
\leq c_{n, k}^{*} a^{n l} b^{k l}\left[\left(\int_{0}^{a} \int_{0}^{b}\left|x_{1}^{(n, k)}(s, t)\right|^{l+m} d s d t\right)^{\frac{l}{m}} \cdot \int_{0}^{a} \int_{0}^{b}\left|x_{2}^{(n, k)}(s, t)\right|^{m+l} d s d t\right. \\
\left.+\left(\int_{0}^{a} \int_{0}^{b}\left|x_{2}^{(n, k)}(s, t)\right|^{l+m} d s d t\right)^{\frac{l}{m}} \cdot \int_{0}^{a} \int_{0}^{b}\left|x_{1}^{(n, k)}(s, t)\right|^{m+l} d s d t\right]^{\frac{m}{l+m}},
\end{gathered}
$$

where $x_{j}^{(n, k)}(s, t)$ is defined in Theorem 1.1, and $c_{n, k}^{*}$ is defined in Theorem 1.2. Proof. From (2.2), we have

$$
\left|x_{1}(s, t)\right| \leq \frac{1}{(n-1) !(k-1) !} \int_{0}^{s} \int_{0}^{t}(s-\sigma)^{n-1}(t-\tau)^{k-1}\left|x_{1}^{(n, k)}(\sigma, \tau)\right| d \sigma d \tau,
$$

by Hölder's inequality with indices $l+m$ and $\frac{l+m}{l+m-1}$, it follows that

$$
\begin{aligned}
\left|x_{1}(s, t)\right| \leq & \frac{1}{(n-1) !(k-1) !}\left(\int_{0}^{s} \int_{0}^{t}\left[(s-\sigma)^{n-1}(t-\tau)^{k-1}\right]^{\frac{l+m}{l+m-1}} d \sigma d \tau\right)^{\frac{l+m-1}{l+m}} \\
& \times\left(\int_{0}^{s} \int_{0}^{t}\left|x_{1}^{(n, k)}(\sigma, \tau)\right|^{l+m} d \sigma d \tau\right)^{\frac{1}{l+m}} \\
= & A s^{n-\xi} t^{k-\xi}\left(\int_{0}^{s} \int_{0}^{t}\left|x_{1}^{(n, k)}(\sigma, \tau)\right|^{l+m} d \sigma d \tau\right)^{\xi},
\end{aligned}
$$

where

$$
A=\left(\frac{(1-\xi)^{2}}{(n-\xi)(k-\xi)}\right)^{1-\xi} \frac{1}{(n-1) !(k-1) !} .
$$

Multiplying the both sides of above inequality by $\left|x_{2}^{(n, k)}(s, t)\right|^{m}$ and integrating both sides over $t$ from 0 to $b$ first and then integrating the resulting inequality over $s$ from 0 to $a$, we obtain

$$
\int_{0}^{a} \int_{0}^{b}\left|x_{1}(s, t)\right|^{l}\left|x_{2}^{(n, k)}(s, t)\right|^{m} d s d t
$$


ON OPIAL INEQUALITIES INVOLVING HIGHER ORDER DERIVATIVES

1271

$$
\leq A^{l} \int_{0}^{a} \int_{0}^{b} s^{l(n-\xi)} t^{l(k-\xi)}\left|x_{2}^{(n, k)}(s, t)\right|^{m}\left(\int_{0}^{s} \int_{0}^{t}\left|x_{1}^{(n, k)}(\sigma, \tau)\right|^{l+m} d \sigma d \tau\right)^{l \xi} d s d t .
$$

Now, applying Hölder's inequality with indices $\frac{l+m}{l}$ and $\frac{l+m}{m}$ to the integral on the right side, we obtain

$$
\begin{aligned}
& \int_{0}^{a} \int_{0}^{b}\left|x_{1}(s, t)\right|^{l}\left|x_{2}^{(n, k)}(s, t)\right|^{m} d s d t \\
\leq & A^{l}\left(\int_{0}^{a} \int_{0}^{b} s^{(n-\xi)(l+m)} t^{(k-\xi)(l+m)} d s d t\right)^{\frac{l}{l+m}} \\
& \times\left(\int_{0}^{a} \int_{0}^{b}\left|x_{2}^{(n, k)}(s, t)\right|^{m+l}\left(\int_{0}^{s} \int_{0}^{t}\left|x_{1}^{(n, k)}(\sigma, \tau)\right|^{l+m} d \sigma d \tau\right)^{\frac{l}{m}} d s d t\right)^{\frac{m}{l+m}} \\
= & A^{l}\left(\frac{\xi^{2}}{n k}\right)^{\xi l} a^{n l} b^{k l} \\
& \times\left(\int_{0}^{a} \int_{0}^{b}\left|x_{2}^{(n, k)}(s, t)\right|^{m+l}\left(\int_{0}^{s} \int_{0}^{t}\left|x_{1}^{(n, k)}(\sigma, \tau)\right|^{l+m} d \sigma d \tau\right)^{\frac{l}{m}} d s d t\right)^{\frac{m}{l+m}} .
\end{aligned}
$$

Similarly, we obtain

$$
\begin{aligned}
& \int_{0}^{a} \int_{0}^{b}\left|x_{2}(s, t)\right|^{l}\left|x_{1}^{(n, k)}(s, t)\right|^{m} d s d t \\
\leq & A^{l}\left(\frac{\xi^{2}}{n k}\right)^{\xi l} a^{n l} b^{k l} \\
& \times\left(\int_{0}^{a} \int_{0}^{b}\left|x_{1}^{(n, k)}(s, t)\right|^{m+l}\left(\int_{0}^{s} \int_{0}^{t}\left|x_{2}^{(n, k)}(\sigma, \tau)\right|^{l+m} d \sigma d \tau\right)^{\frac{l}{m}} d s d t\right)^{\frac{m}{l+m}} .
\end{aligned}
$$

Thus, from $(2.12),(2.13)$ and in view of the elementary inequality $a^{\lambda}+b^{\lambda} \leq$ $2^{1-\lambda}(a+b)^{\lambda}, 0 \leq \lambda \leq 1, a, b \geq 0$, we obtain

$$
\begin{aligned}
& \int_{0}^{a} \int_{0}^{b}\left[\left|x_{1}(s, t)\right|^{l}\left|x_{2}^{(n, k)}(s, t)\right|^{m}+\left|x_{2}(s, t)\right|^{l}\left|x_{1}^{(n, k)}(s, t)\right|^{m}\right] d s d t \\
\leq & c_{n, k}^{*} a^{n l} b^{k l}\left[\int_{0}^{a} \int_{0}^{b}\left|x_{2}^{(n, k)}(s, t)\right|^{m+l}\left(\int_{0}^{s} \int_{0}^{t}\left|x_{1}^{(n, k)}(\sigma, \tau) d \sigma d \tau\right|\right)^{\frac{l}{m}}\right. \\
& \left.+\int_{0}^{a} \int_{0}^{b}\left|x_{1}^{(n, k)}(s, t)\right|^{m+l}\left(\int_{0}^{s} \int_{0}^{t}\left|x_{2}^{(n, k)}(\sigma, \tau)\right|^{l+m} d \sigma d \tau\right)^{\frac{l}{m}} d s d t\right]^{\frac{m}{l+m}}
\end{aligned}
$$




$$
\begin{aligned}
\leq & c_{n, k}^{*} a^{n l} b^{k l}\left[\left(\int_{0}^{a} \int_{0}^{b}\left|x_{1}^{(n, k)}(s, t)\right|^{l+m} d s d t\right)^{\frac{l}{m}} \cdot \int_{0}^{a} \int_{0}^{b}\left|x_{2}^{(n, k)}(s, t)\right|^{m+l} d s d t\right. \\
& \left.+\left(\int_{0}^{a} \int_{0}^{b}\left|x_{2}^{(n, k)}(s, t)\right|^{l+m} d s d t\right)^{\frac{l}{m}} \cdot \int_{0}^{a} \int_{0}^{b}\left|x_{1}^{(n, k)}(s, t)\right|^{m+l} d s d t\right]^{\frac{m}{l+m}} .
\end{aligned}
$$

This completes the proof.

Remark 2.6. Taking for $x_{1}(s, t)=x_{2}(s, t)=x(s, t)$ in (2.11), we have

$$
\int_{0}^{a} \int_{0}^{b}|x(s, t)|^{l} \cdot\left|x^{(n, k)}(s, t)\right|^{m} d s d t \leq c_{n, k}^{*} a^{n l} b^{k l} \int_{0}^{a} \int_{0}^{b}\left|x^{(n, k)}(s, t)\right|^{l+m} d s d t .
$$

Remark 2.7. Let $x(s, t)$ reduce to $x(t)$ by letting $s=s(t)$ and with suitable modifications in all intermediate steps of proof of Theorem 2.5, then (2.11) becomes the following result.

$$
\begin{aligned}
& \int_{0}^{a}\left[\left|x_{1}(t)\right|^{l}\left|x_{2}^{(n)}(t)\right|^{m}+\left|x_{2}(t)\right|^{l}\left|x_{1}^{(n)}(t)\right|^{m}\right] d t \\
\leq & c_{n}^{*} a^{n l}\left[\left(\int_{0}^{a}\left|x_{1}^{(n)}(t)\right|^{l+m} d t\right)^{\frac{l}{m}} \cdot \int_{0}^{a}\left|x_{2}^{(n)}(t)\right|^{m+l} d t\right. \\
& \left.+\left(\int_{0}^{a}\left|x_{2}^{(n)}(t)\right|^{l+m} d t\right)^{\frac{l}{m}} \cdot \int_{0}^{a}\left|x_{1}^{(n, k)}(t)\right|^{m+l} d t\right]^{\frac{m}{l+m}},
\end{aligned}
$$

where

$$
c_{n}^{*}=\left(\frac{n(1-\xi)}{n-\xi}\right)^{l(1-\xi)} \frac{2^{\xi l} \xi^{\xi l}}{(n !)^{l}}, \xi=\frac{1}{l+m} .
$$

Taking for $x_{1}(t)=x_{2}(t)=x(t)$ in (2.14), we have the following interesting inequality.

$$
\int_{0}^{a}|x(t)|^{l}\left|x^{(n)}(t)\right|^{m} d t \leq c_{n}^{*} a^{n l} \int_{0}^{a}\left|x^{(n)}(t)\right|^{l+m} d t .
$$

Acknowledgements. The authors express their grateful thanks to the referee for his many valuable help.

\section{References}

[1] R. P. Agarwal, Sharp Opial-type inequalities involving r-derivatives and their applications, Tohoku Math. J. 47 (1995), no. 4, 567-593.

[2] R. P. Agarwal and V. Lakshmikantham, Uniqueness and Nonuniqueness Criteria for Ordinary Differential Equations, World Scientific, Singapore, 1993.

[3] R. P. Agarwal and P. Y. H. Pang, Opial Inequalities with Applications in Differential and Difference Equations, Kluwer Academic Publishers, Dordrecht, 1995.

[4] - Sharp Opial-type inequalities in two variables, Appl. Anal. 56 (1995), no. 3-4, $227-242$.

[5] R. P. Agarwal and E. Thandapani, On some new integro-differential inequalities, Anal. sti. Univ. "Al. I. Cuza" din Iasi 28 (1982), no. 1, 123-126. 
[6] H. Alzer, An Opial-type inequality involving higher-order derivatives of two functions, Appl. Math. Lett. 10 (1997), no. 4, 123-128.

[7] D. Bainov and P. Simeonov, Integral Inequalities and Applications, Kluwer Academic Publishers, Dordrecht, 1992.

[8] P. R. Beesack, On an integral inequality of Z. Opial, Trans. Amer. Math. Soc. 104 (1962), 470-475.

[9] W. S. Cheung, On Opial-type inequalities in two variables, Aequationes Math. 38 (1989), no. 2-3, 236-244.

[10] _ Some new Opial-type inequalities, Mathematika 37 (1990), no. 1, 136-142.

[11] Some generalized Opial-type inequalities, J. Math. Anal. Appl. 162 (1991), no. $2,317-321$.

[12] _ Opial-type inequalities with $m$ functions in $n$ variables, Mathematika 39 (1992), no. 2, 319-326.

[13] W. S. Cheung, D. D. Zhao, and J. E. Pečarić, Opial-type inequalities for Differential Operators, to appear in Nonlinear Anal.

[14] K. M. Das, An inequality similar to Opial's inequality, Proc. Amer. Math. Soc. 22 (1969), 258-261.

[15] E. K. Godunova and V. I. Levin, An inequality of Maroni, Mat. Zametki 2 (1967), $221-224$.

[16] L. K. Hua, On an inequality of Opial, Sci. Sinica 14 (1965), 789-790.

[17] B. Karpuz, B. Kaymakcalan, and U. M. Özkan, Some multi-dimenstonal Opial-type inequalities on time scales, J. Math. Inequal. 4 (2010), no. 2, 207-216.

[18] J. D. Li, Opial-type integral inequalities involving several higher order derivatives, J. Math. Anal. Appl. 167 (1992), no. 1, 98-100.

[19] D. S. Mitrinovič, Analytic Inequalities, Springer-Verlag, Berlin, New York, 1970.

[20] D. S. Mitrinovič, J. E. Pečarić, and A. M. Fink, Inequalities involving Functions and Their Integrals and Derivatives, Kluwer Academic Publishers, Dordrecht, 1991.

[21] Z. Opial, Sur une inégalité, Ann. Polon. Math. 8 (1960), 29-32.

[22] B. G. Pachpatte, On integral inequalities similar to Opial's inequality, Demonstratio Math. 22 (1989), no. 1, 21-27.

[23] _ On Opial-type integral inequalities, J. Math. Anal. Appl. 120 (1986), no. 2, $547-556$.

[24] - On some new generalizations of Opial inequality, Demonstratio Math. 19 (1986), no. 2, 281-291.

[25] A note on generalized Opial type inequalities, Tamkang J. Math. 24 (1993), no. $2,229-235$.

[26] J. E. Pečarić, An integral inequality, Analysis, geometry and groups: a Riemann legacy volume, 471-478, Hadronic Press Collect. Orig. Artic., Hadronic Press, Palm Harbor, FL, 1993.

[27] J. E. Pečarić and I. Brnetić, Note on generalization of Godunova-Levin-Opial inequality, Demonstratio Math. 30 (1997), no. 3, 545-549.

[28] Note on the generalization of the Godunova-Levin-Opial inequality in several independent variables, J. Math. Anal. Appl. 215 (1997), no. 1, 274-282.

[29] G. I. Rozanova, Integral inequalities with derivatives and with arbitrary convex functions, Moskov. Gos. Ped. Inst. Vcen. Zap. 460 (1972), 58-65.

[30] D. Willett, The existence-uniqueness theorem for an $n$-th order linear ordinary differential equation, Amer. Math. Monthly 75 (1968), 174-178.

[31] G. S. Yang, Inequality of Opial-type in two variables, Tamkang J. Math. 13 (1982), no. $2,255-259$.

[32] - On a certain result of Z. Opial, Proc. Japan Acad. 42 (1966), 78-83.

[33] C. J. Zhao and W. S. Cheung, Sharp integral inequalities involving high-order partial derivatives, J. Ineq. Appl. 2008 (2008), Article ID 571417, 10 pages. 
CHANG-JiAN ZHAO

Department of Mathematics

China Jiliang University

Hangzhou 310018, P. R. China

E-mail address: chjzhao@163.com; chjzhao@yahoo.com.cn

Wing-Sum Cheung

Department of Mathematics

The University of Hong Kong

Pokfulam Road, Hong Kong

E-mail address: wscheung@hku.hk 